\title{
Gapless Spin Excitations in the Field-Induced Quantum Spin Liquid Phase of $\alpha-\mathrm{RuCl}_{3}$
}

\author{
Jiacheng Zheng, ${ }^{1,2}$ Kejing Ran, ${ }^{3}$ Tianrun Li, ${ }^{1}$ Jinghui Wang, ${ }^{3}$ Pengshuai Wang, ${ }^{1}$ Bin Liu, ${ }^{2}$ Zheng-Xin Liu, ${ }^{1}$ \\ B. Normand, ${ }^{4}$ Jinsheng Wen, ${ }^{3,5, *}$ and Weiqiang $\mathrm{Yu}^{1,6, *}$ \\ ${ }^{1}$ Department of Physics and Beijing Key Laboratory of Opto-electronic Functional Materials \& Micro-nano Devices, \\ Renmin University of China, Beijing 100872, China \\ ${ }^{2}$ Department of Physics, Beijing Jiaotong University, Beijing 100044, China \\ ${ }^{3}$ National Laboratory of Solid State Microstructures and Department of Physics, Nanjing University, \\ Nanjing 210093, China \\ ${ }^{4}$ Laboratory for Neutron Scattering and Imaging, Paul Scherrer Institute, CH-5232 Villigen-PSI, Switzerland \\ ${ }^{5}$ Innovative Center for Advanced Microstructures, Nanjing University, Nanjing 210093, China \\ ${ }^{6}$ Department of Physics and Astronomy, Shanghai Jiao Tong University, Shanghai 200240, China \\ (Received 24 March 2017; revised manuscript received 14 August 2017; published 1 December 2017)
}

$\alpha-\mathrm{RuCl}_{3}$ is a leading candidate material for the observation of physics related to the Kitaev quantum spin liquid (QSL). By combined susceptibility, specific-heat, and nuclear-magnetic-resonance measurements, we demonstrate that $\alpha-\mathrm{RuCl}_{3}$ undergoes a quantum phase transition to a QSL in a magnetic field of $7.5 \mathrm{~T}$ applied in the $a b$ plane. We show further that this high-field QSL phase has gapless spin excitations over a field range up to $16 \mathrm{~T}$. This highly unconventional result, unknown in either Heisenberg or Kitaev magnets, offers insight essential to establishing the physics of $\alpha-\mathrm{RuCl}_{3}$.

DOI: $10.1103 /$ PhysRevLett.119.227208

The quantum spin liquid (QSL) is an exotic state of matter with long-range coherence but with no spontaneous breaking of translational or spin-rotational symmetry down to zero temperature [1]. Such a state in two or higher dimensions has implications for phenomena ranging from high-temperature superconductivity [2] to quantum computation $[3,4]$. QSLs have long been sought in systems with strong geometric frustration [5-8], where magnetic order is destroyed by quantum fluctuations in a highly degenerate ground manifold. A more recent avenue to QSL formation is by competing interactions with combined spin and spatial anisotropies, as in the Kitaev model, where both gapped and gapless QSLs are realized exactly in a honeycomb-lattice spin-1/2 system [9].

A pure Kitaev Hamiltonian is hard to achieve in real materials. However, the compounds $A_{2} \operatorname{IrO}_{3}(A=\mathrm{Na}, \mathrm{Li})$ [10-15] and $\alpha-\mathrm{RuCl}_{3}$ [16-22] are candidate systems for significant Kitaev-type interactions. In each case, the $4 d$ $\left(\mathrm{Ru}^{3+}\right)$ or $5 d\left(\mathrm{Ir}^{4+}\right)$ ions form a Mott insulator on a honeycomb lattice, whose localized electrons have an effective spin $j_{\text {eff }}=1 / 2$ due to strong spin-orbit coupling [10,23-26]. In $\alpha-\mathrm{RuCl}_{3}$ at zero field, a finite-energy continuum of magnetic excitations [27] is suggestive of fractionalized (spinon or Majorana-fermion) excitations [9,28-32]. However, the ground states in all cases have "zigzag" magnetic order [33-38], indicating the presence of significant non-Kitaev terms, whose exact nature continues to occupy many authors [39-48]. While the large $T_{N}$ in $\mathrm{Na}_{2} \mathrm{IrO}_{3}$ [33,34] suggests subdominant Kitaev terms, the relatively low $T_{N}$ of $\alpha-\mathrm{RuCl}_{3}$ has sparked an intensive search for experimental [49-51] and theoretical [52,53] evidence for "proximate Kitaev" behavior.
Here we report a nuclear-magnetic-resonance (NMR) investigation of high-quality single crystals of $\alpha-\mathrm{RuCl}_{3}$. With additional magnetic susceptibility and specific-heat measurements, we establish the phase diagram of Fig. 1. We demonstrate the presence of a field-induced QSL beyond the quantum phase transition at $\mu_{0} H_{c} \simeq 7.5 \mathrm{~T}$. In the field range between 7.5 and $16 \mathrm{~T}$, this partially polarized QSL has a spin-lattice relaxation rate with

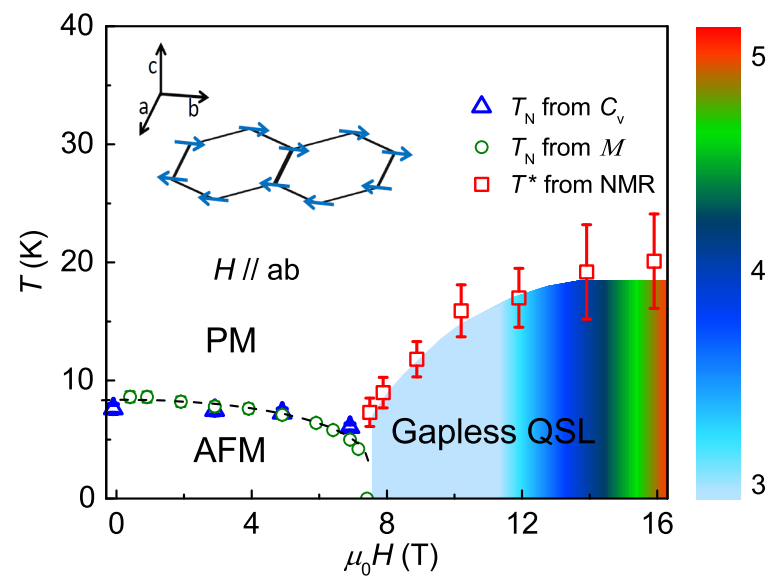

FIG. 1. Magnetic phase diagram of $\alpha-\mathrm{RuCl}_{3}$ with field applied in the $a b$ plane. $T_{N}$ is determined from magnetization and specific-heat data (Fig. 2). In the QSL phase, the color map represents the exponent, $\alpha$, determined from the power-law form of the NMR spin-lattice relaxation rate, $1 /{ }^{35} T_{1} \propto T^{\alpha}$ (Fig. 4). $T^{*}$ represents the upper limit of the gapless low- $T$ regime. Inset: schematic representation of zero-field zigzag order in the hexagonal $(a b)$ plane. 

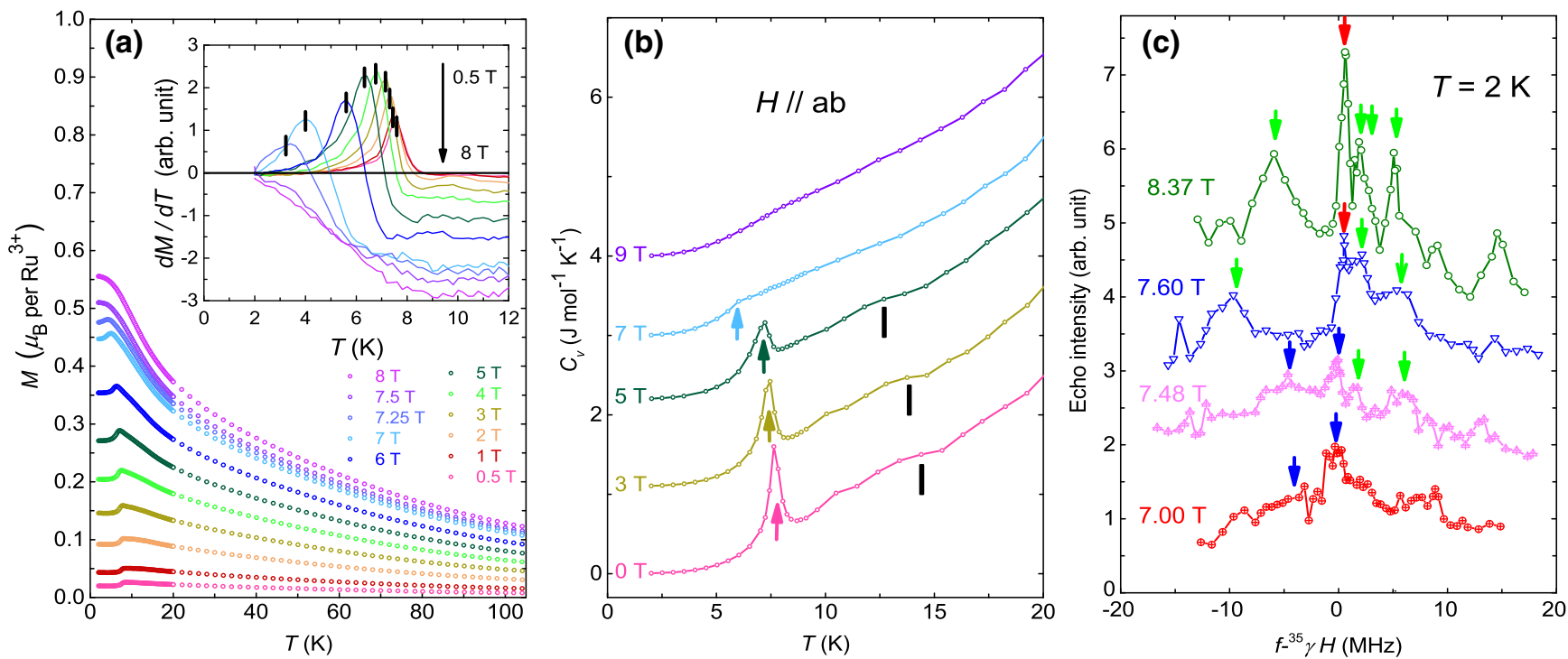

FIG. 2. Magnetic transition with field applied in the $a b$ plane. (a) Magnetization, $M(T)$. Inset: $d M / d T$; vertical lines mark the peaks, which show $T_{N}$ for fields up to $7.25 \mathrm{~T}$. (b) Low- $T$ specific heat, $C_{v}$. Data are offset for clarity. The transition for regions with $A B C$ ( $A B$ ) layer stacking is marked by the arrows (vertical lines). (c) ${ }^{35} \mathrm{Cl}$ NMR spectra at fields close to $7.5 \mathrm{~T}$, shown at $T=2 \mathrm{~K}$. Blue arrows mark peaks characteristic of the low-field, ordered phase, green arrows the peaks of the high-field, disordered phase, and red arrows the sharp central peak at which ${ }^{35} K_{n}$ and $1 /{ }^{35} T_{1}$ were measured.

power-law temperature dependence, indicating effectively gapless spin excitations with line-node dispersion.

Single crystals of $\alpha-\mathrm{RuCl}_{3}$ were grown by chemical vapor transport. The high quality of this batch of crystals is demonstrated in Ref. [54] and x-ray characterization of the NMR sample, shown in Sec. S1 of the Supplemental Material [55], demonstrated that it is a single domain and free of twinning. Magnetization measurements were performed in a 9 T SQUID and the specific heat measured in a Quantum Design PPMS. ${ }^{35} \mathrm{Cl}$ NMR spectra were collected by the spin-echo technique and the spin-lattice relaxation rate measured by the inversion-recovery method, as shown in Sec. S2 of the Supplemental Material [55]. The spin-recovery exponent, $\beta=1$ in the paramagnetic state $(T>20 \mathrm{~K}$ in Fig. 1), also indicates a very high sample quality.

The magnetization $M(T)$ is shown for a range of applied fields in Fig. 2(a), with its primary features, emphasized by the low- $T$ derivative $d M / d T$ shown in the inset. At zero field, $\alpha-\mathrm{RuCl}_{3}$ has zigzag antiferromagnetic (AFM) order (inset, Fig. 1) below $T_{N} \simeq 7.5 \mathrm{~K}(14 \mathrm{~K}$ ) for crystals with $A B C(A B)$ stacking along the $c$ axis [38]. The sharp phase transition at $7.5 \mathrm{~K}$ in both $d M / d T$ and the specific heat $C_{v}$ [Fig. 2(b)] demonstrates the very high crystal quality. Our samples are almost exclusively $A B C$ stacked, with only a small admixture of $A B$ stacking discernible through the weak anomaly in $C_{v}$. Our $C_{v}$ data include the phonon contribution; due to concerns over its subtraction and over the suitability of specific heat for this purpose, we do not attempt to use our data to analyze the magnetic response.
Fields applied in the $a b$ plane suppress $T_{N}$. This effect is especially strong from 7 to $7.25 \mathrm{~T}$, leading up to a fieldinduced quantum phase transition (QPT) at $\mu_{0} H_{c} \simeq 7.5 \mathrm{~T}$. The QPT is observed clearly both in the disappearance of the peaks in $d M / d T$ and $C_{v}$ and in the dramatic changes in the NMR spectra [Fig. 2(c)], which we discuss next. We find no anisotropy in $M$ and $C_{v}$ as the field is rotated in the $a b$ plane. The NMR measurements shown in Fig. 2(c), and also Fig. 3, are fully representative of the generic in-plane response, as we demonstrate in Secs. S3 and S4 of the Supplemental Material [55].

We collect the evidence that the phase at $H>H_{c}$ is a QSL. First, the vanishing peaks in both $d M / d T$ and $C_{v}$ (Fig. 2) demonstrate the absence of magnetic order. Second, the ${ }^{35} \mathrm{Cl}$ line shapes for fields at and above 7.6 $\mathrm{T}$ contain none of the peaks corresponding to an ordered $\mathrm{Cl}$ environment. The ${ }^{35} \mathrm{Cl} \mathrm{NMR}$ spectra below 7.6 T [Fig. 2(c)] are very broad at low $T$, consistent in the absence of domain and twinning effects only with AFM order. By contrast, several sharp peaks are clearly resolvable for fields above $7.6 \mathrm{~T}$ in Figs. 2(c), 3(a), and 3(b). The center peak at $\Delta f \approx 0(\gamma H \approx 43.5 \mathrm{MHz})$ has a FWHM height of $0.5 \mathrm{MHz}$ at $10.3 \mathrm{~T}$ at both $T=20$ and $2 \mathrm{~K}$ [Fig. 3(a)], and shows no significant changes at $12 \mathrm{~T}$ [Fig. 3(b)]. Such narrow linewidths, and, in particular, their invariance upon cooling below $20 \mathrm{~K}$, indicate a complete absence of magnetic order.

Further evidence is provided by the Knight shift ${ }^{35} K_{n}$ of the center peak, shown in Fig. 3(c). From its large values $(6 \%-7 \%)$ below $10 \mathrm{~K}$, the hyperfine field is strong at the ${ }^{35} \mathrm{Cl}$ site and magnetic order, if present, is very unlikely to 

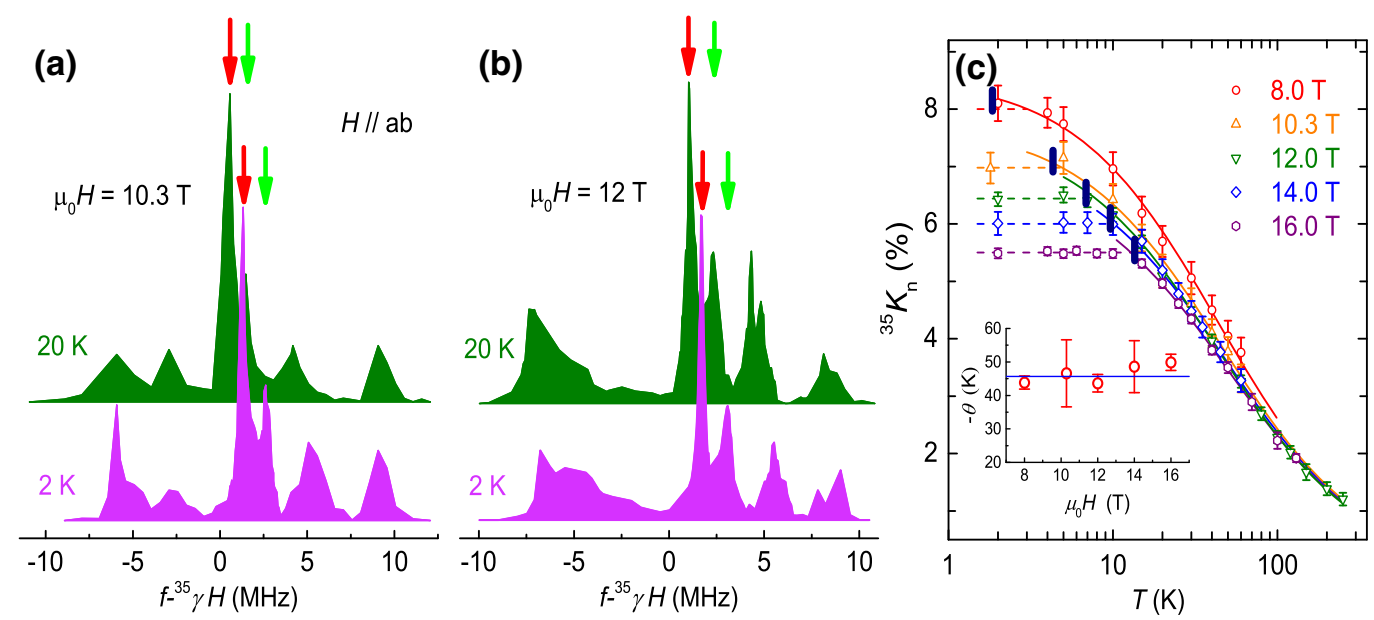

FIG. 3. ${ }^{35} \mathrm{Cl}$ NMR spectra and Knight shift for in-plane fields. ${ }^{35} \mathrm{Cl}$ spectra measured at (a) 10.3 and (b) $12 \mathrm{~T}$, shown for $T=2$ and $20 \mathrm{~K} . \Delta f=f-{ }^{35} \gamma \mathrm{H}$ is the frequency shift, where $f$ is the measured frequency and ${ }^{35} \gamma=4.171 \mathrm{MHz} / \mathrm{T}$ is the gyromagnetic ratio of ${ }^{35} \mathrm{Cl}$. The splitting of the sharp central peak (red and green arrows) is a consequence of the three inequivalent $\mathrm{Cl}^{-}$sites, two of which are not separated at this field angle. The separation of the broader satellite peaks from the center peaks changes little with field. The peak widths are determined by quadrupolar effects [56], discussed in Sec. S5 of the Supplemental Material [55]. (c) NMR Knight shift, ${ }^{35} K_{n}(T)$, measured at the central peak and shown for different field values. ${ }^{35} K_{n}$ is calculated from $\Delta f$ after subtracting all $T$-independent quadrupolar and orbital contributions (Sec. S5 [55]). Solid lines are Curie-Weiss fits to the high- $T$ data, of the form ${ }^{35} K_{n}=a /(T-\theta)$; we find that $\theta=-45 \pm 10 \mathrm{~K}$ is almost field independent (inset). Dotted lines indicate a "level-off" behavior of ${ }^{35} K_{n}$ at low temperatures (marked by the vertical lines).

be missed by NMR. This statement remains true even for incommensurate or large-unit-cell ordered phases that are possible in a field [52]. Further, a Curie-Weiss (CW) fit to the high-temperature part of the data holds down to $2 \mathrm{~K}$ at fields of $8 \mathrm{~T}$, with the $\mathrm{CW}$ temperature $\theta \approx-45 \mathrm{~K}$ [inset, Fig. 3(c)], supporting the absence of magnetic order. Because the NMR spectra show no evidence that the magnetic QPT is accompanied by a structural transition, which would cause much more dramatic peak shifts in Fig. 2(c), we conclude that the field-induced disordered phase is indeed a QSL.

Unlike $T_{N}$, the value of $H_{c}$ required to suppress magnetic order in a crystal with $A B$ stacking is also around $8 \mathrm{~T}$ [37]. The similarity of $H_{c}$ values for $A B C$ and $A B$ stacking suggests that the field-induced suppression of magnetic order, and by extension the properties of the highfield QSL phase, are primarily two-dimensional (2D) in nature, rather than depending on interlayer coupling.

The spin excitations of the high-field QSL state are probed by the spin-lattice relaxation rate. We measure $1 /{ }^{35} T_{1}$ for each field at the sharp central peak marked by the red arrows in Figs. 2 and 3, and show the results in Fig. 4. Above $40 \mathrm{~K}, 1 /{ }^{35} T_{1}$ remains constant in both $T$ and $H$, which is typical of decorrelated spins at temperatures beyond their interaction energy scale. At $8 \mathrm{~T}, 1 /{ }^{35} T_{1}$ drops sharply on cooling below $10 \mathrm{~K}$, and follows a power-law $T$ dependence, $1 /{ }^{35} T_{1}=b T^{\alpha}$, to the bottom of our measurement range $(T=1.5 \mathrm{~K})$. The same behavior holds at all fields up to our maximum of $16 \mathrm{~T}$. The exponent $\alpha$, shown in the inset of Fig. 4, remains constant at $\alpha=3$ for fields between 8 and $12 \mathrm{~T}$, and then increases to $\alpha=5$ at $16 \mathrm{~T}$. While exponents above 5 suggest the opening of a full gap, the reliable extraction of power-law behavior with exponents as small as $\alpha=3$ demonstrates that the QSL in the field range $8<\mu_{0} H<16 \mathrm{~T}$ has either gapless spin excitations or an anomalously small gap far below $2 \mathrm{~K}$.

We extract also the characteristic temperature $T^{*}$ marking the upper limit of the low- $T$, power-law regime, which is shown in Fig. 4 and also in Fig. 1. The increase of $T^{*}$ with field demonstrates that the phase of coherent QSL dynamics becomes increasingly robust, at least to $16 \mathrm{~T}$. This occurs in tandem with an increasing field-driven polarization, observed in both $M$ and ${ }^{35} K_{n}$, which leaves a decreasing component of the spin available to participate in the QSL. The related average moment per Ru ion has been measured directly [37] as $0.56 \mu_{B}$ at $8,0.87 \mu_{B}$ at 16 , and $1.22 \mu_{B}$ at $60 \mathrm{~T}$. For comparison, the static moment in the zigzag AFM state at $H=0$ is $0.4 \mu_{B}$. We note that the recovery exponent $\beta$ decreases at $T<T^{*}$, suggesting that the QSL dynamics are very sensitive to any weak disorder. However, as discussed in Sec. S2 of the Supplemental Material [55], our results contain no evidence that the QSL state itself could be a consequence of disorder, and verify rather its intrinsic nature.

Two recent experiments have also investigated $\alpha-\mathrm{RuCl}_{3}$ in fields exceeding $H_{c}$. Thermal conductivity measurements show a prominent low- $T$ peak whose magnitude grows linearly with $H-H_{c}$ [50], indicating a gapless and linearly dispersive excitation. By contrast, a different NMR study reports gapped behavior [51], although 


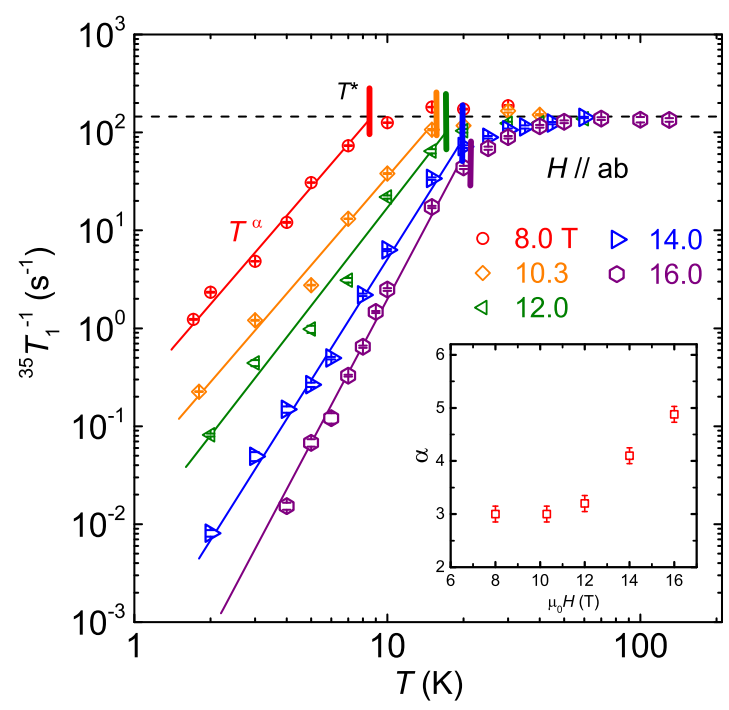

FIG. 4. NMR spin-lattice relaxation rates. $1 /{ }^{35} T_{1}$ shown for five different applied in-plane fields. At $T>40 \mathrm{~K}, 1 /{ }^{35} T_{1}$ approaches a constant value, $1 /{ }^{35} T_{1} \simeq 145 \mathrm{~s}^{-1}$, for all fields (dashed line). At low $T, 1 /{ }^{35} T_{1}$ follows a power-law $T$ dependence; solid lines are fits to the form $1 /{ }^{35} T_{1}=b T^{\alpha}$ over one decade in $T$. The exponent $\alpha$ (inset) and temperature scale $T^{*}$ (vertical lines) are also shown in Fig. 1.

the field is applied at an angle $30^{\circ}$ out of the $a b$ plane. There is increasing evidence that different field orientations lead to qualitatively different properties in $\alpha-\mathrm{RuCl}_{3}$, as expected from the strong spin anisotropy (our own NMR investigation is presented in Sec. S4 of the Supplemental Material [55]). However, the gaps reported by these authors are deduced only over a limited temperature range that, crucially, does not extend to $T=0$, and the finite $1 / T_{1}$ observed at low $T$ is not consistent with a spin gap.

To interpret our results, we consider the definition $1 / T_{1} T=\lim _{\omega \rightarrow 0} \sum_{q} A_{\mathrm{hf}}(q) \operatorname{Im} \chi(q, \omega) / \omega$, where $\chi(q, \omega)$ is the dynamical susceptibility and $A_{\mathrm{hf}}(q)$ the hyperfine coupling. For a conventional magnetic system, this may be reexpressed as $1 / T_{1} \sim \int_{0}^{\infty} \rho^{2}(E) n(E)[1+n(E)] d E$, where $\rho(E)$ is the magnon density of states (DOS) and $n(E)$ the Bose distribution function, and this makes $1 / T_{1}$ a sensitive probe of low-energy spin dynamics. Our result $1 / T_{1} \sim T^{3}$ suggests that the density of magnetic states follows $\rho(E) \propto E$. In condensed matter, such a linear DOS is more familiar from fermionic excitations, including line nodes in the gap at the Fermi surface of a 3D superconductor [57] and the 2D Dirac-cone dispersion [58].

In conventional (Heisenberg) quantum magnets, the partial polarization is distributed uniformly and becomes complete at a finite saturation field, $H_{s}$. All spin excitations are bosonic and for $H>H_{s}$ they have a gap $\Delta \propto H-H_{s}$. Spatially anisotropic and frustrated systems show magnetization plateaus at low or intermediate fields, whose ground states are in general gapped [59] with bosonic excitations. Thus our discovery of gapless spin excitations at intermediate fields in $\alpha-\mathrm{RuCl}_{3}$ represents an extremely unconventional situation.

When a Hamiltonian anisotropic in spin space does not commute with the field, $H_{s} \rightarrow \infty$. Although the spatially isotropic Kitaev model has one gapless and linearly dispersive Majorana fermion at zero field, its spin excitations, consisting of Majorana fermions coupled to two massive, static flux quanta [9,29], are gapped. In Ref. [60] it was shown that the spin gap can vanish in a "generic Kitaev" system, meaning one in which the symmetryallowed Heisenberg $(J)$ and off-diagonal $(\Gamma)$ terms are present at a perturbative level, and these authors obtained the highly suggestive result $1 / T_{1} \propto T^{3}$. However, in an applied field, all the modes in both the pure $(K)$ and generic (perturbative $K-J-\Gamma$ ) Kitaev models become gapped. Although a gapless Majorana quasiparticle could be responsible for the measured thermal conductivity [50], our NMR results show unequivocally that the spin excitations themselves are gapless, and thus such proximate Kitaev physics appears to be excluded.

We review scenarios allowing gapless spin excitations over a range of field strengths. As a result of its weak interplane interactions, $\alpha-\mathrm{RuCl}_{3}$ is effectively a $2 \mathrm{D}$ magnet [27] and, therefore, its line nodes are interplane, connecting point nodes in the honeycomb layers. Bosonic excitations in (and beyond) 2D are either gapped or condense, leading to magnetic order, whereas gapless and disordered (i.e., critical) behavior over a finite field range is unknown. Turning to fermionic excitations, these imply a fractionalization and deconfinement of spinonic quasiparticles taking place at the QPT. In the Heisenberg chain and ladder (1D), gapless spinons appear at incommensurate wave vectors over a range of fields. In $2 \mathrm{D}$, some spin-orbitcoupled magnetic systems may be represented by fermionic spinons, whose dispersion has Dirac cones. These cones provide the linear DOS and remain stable, i.e., pinned at the Fermi level, over a finite range of fields applied in specific symmetry directions, whereas fields in generic directions open a gap (an example is shown in Sec. S6 of the Supplemental Material [55]). For Majorana fermions, at least two flavors of Majorana cone are required for the system to host gapless spin excitations.

Concerning a microscopic model exhibiting such exotic physics, the effective magnetic Hamiltonian of the spinorbit-coupled Mott insulator has focused attention on $K-J-\Gamma$-type models $[42,43]$. Opinions on the terms and parameters describing $\alpha-\mathrm{RuCl}_{3}$ remain strongly divergent. Early efforts using a $J-K$ model with ferromagnetic $J$ and AFM $K$ [27] have been supplanted by an exchange of signs and longer-ranged $J$ terms $[45,53]$. Very recent studies $[48,49,54]$ have turned to $K-\Gamma$ models with parameters far outside the perturbative regime of Ref. [60]. Although the effects of a magnetic field have yet to be investigated 
in detail, our own $K-\Gamma$ analysis ([61], Sec. S6 of the Supplemental Material [55]) indicates robust QSL states whose gapless fermionic spinons have four dispersion cones for certain field directions and a very small gap for all in-plane fields.

In summary, we have observed a QPT to a field-induced QSL above $\mu_{0} H_{c}=7.5 \mathrm{~T}$ in $\alpha-\mathrm{RuCl}_{3}$. We have shown by NMR measurements that this state has effectively gapless spin excitations over a broad field range. This result cannot be reconciled with the behavior of conventional quantum magnets or of the pure or generic Kitaev QSL. Thus, our data suggest fractionalized spinon excitations in $\alpha-\mathrm{RuCl}_{3}$ and set a significant challenge to theory.

This work was supported by the Ministry of Science and Technology of China (Grant No. 2016YFA0300504), by the National Natural Science Foundation of China (Grants No. 11374364, No. 11374143, No. 11574392, No. 11674157, and No. 11774025), and by the Fundamental Research Funds for the Central Universities and the Research Funds of Renmin University of China (Grant No. 14XNLF08).

J. C. Z. and K. J. R. contributed equally to this study.

*jwen@nju.edu.cn

†qyu_phy@ruc.edu.cn

[1] L. Balents, Nature (London) 464, 199 (2010).

[2] P. W. Anderson, Science 235, 1196 (1987).

[3] A. Yu. Kitaev, Ann. Phys. (Amsterdam) 303, 2 (2003).

[4] C. Nayak, S. H. Simon, A. Stern, M. Freedman, and S. Das Sarma, Rev. Mod. Phys. 80, 1083 (2008).

[5] P. W. Anderson, Mater. Res. Bull. 8, 153 (1973).

[6] Y. Shimizu, K. Miyagawa, K. Kanoda, M. Maesato, and G. Saito, Phys. Rev. Lett. 91, 107001 (2003).

[7] T.-H. Han, J. S. Helton, S. Y. Chu, D. G. Nocera, J. A. Rodriguez-Rivera, C. Broholm, and Y.S. Lee, Nature (London) 492, 406 (2012).

[8] J. S. Gardner, M. J. P. Gingras, and J. E. Greedan, Rev. Mod. Phys. 82, 53 (2010).

[9] A. Kitaev, Ann. Phys. (Amsterdam) 321, 2 (2006).

[10] G. Jackeli, and G. Khaliullin, Phys. Rev. Lett. 102, 017205 (2009).

[11] J. Chaloupka, G. Jackeli, and G. Khaliullin, Phys. Rev. Lett. 105, 027204 (2010).

[12] Y. Singh, S. Manni, J. Reuther, T. Berlijn, R. Thomale, W. $\mathrm{Ku}, \mathrm{S}$. Trebst, and P. Gegenwart, Phys. Rev. Lett. 108, 127203 (2012).

[13] H. Gretarsson, J. P. Clancy, X. Liu, J. P. Hill, E. Bozin, Y. Singh, S. Manni, P. Gegenwart, J. Kim, A. H. Said, D. Casa, T. Gog, M. H. Upton, H.-S. Kim, J. Yu, V. M. Katukuri, L. Hozoi, J. van den Brink, and Y.-J. Kim, Phys. Rev. Lett. 110, 076402 (2013).

[14] H. S. Chun, J.-W. Kim, J. Kim, H. Zheng, C. C. Stoumpos, C. D. Malliakas, J. F. Mitchell, K. Mehlawat, Y. Singh, Y. Choi, T. Gog, A. Al-Zein, M. M. Sala, M. Krisch, J. Chaloupka, G. Jackeli, G. Khaliullin, and B. J. Kim, Nat. Phys. 11, 462 (2015).
[15] J. G. Rau, E. K. -H. Lee, and H.-Y. Kee, Annu. Rev. Condens. Matter Phys. 7, 195 (2016).

[16] I. Pollini, Phys. Rev. B 53, 12769 (1996).

[17] K. W. Plumb, J. P. Clancy, L. J. Sandilands, V. V. Shankar, Y. F. Hu, K. S. Burch, H.-Y. Kee, and Y.-J. Kim, Phys. Rev. B 90, 041112 (2014).

[18] H.-S. Kim, V. V. Shankar, A. Catuneanu, and H.-Y. Kee, Phys. Rev. B 91, 241110 (2015).

[19] L. J. Sandilands, Y. Tian, K. W. Plumb, Y.-J. Kim, and K. S. Burch, Phys. Rev. Lett. 114, 147201 (2015).

[20] Y. Kubota, H. Tanaka, T. Ono, Y. Narumi, and K. Kindo, Phys. Rev. B 91, 094422 (2015).

[21] L. J. Sandilands, Y. Tian, A. A. Reijnders, H.-S. Kim, K. W. Plumb, Y.-J. Kim, H.-Y. Kee, and K. S. Burch, Phys. Rev. B 93, 075144 (2016).

[22] A. Koitzsch, C. Habenicht, E. Müller, M. Knupfer, B. Büchner, H. C. Kandpal, J. van den Brink, D. Nowak, A. Isaeva, and T. Doert, Phys. Rev. Lett. 117, 126403 (2016).

[23] B. J. Kim, H. Ohsumi, T. Komesu, S. Sakai, T. Morita, H. Takagi, and T. Arima, Science 323, 1329 (2009).

[24] K. Foyevtsova, H. O. Jeschke, I. I. Mazin, D. I. Khomskii, and R. Valentí, Phys. Rev. B 88, 035107 (2013).

[25] J. Chaloupka and G. Khaliullin, Phys. Rev. B 94, 064435 (2016).

[26] T. Birol and K. Haule, Phys. Rev. Lett. 114, 096403 (2015).

[27] A. Banerjee, C. A. Bridges, J. Q. Yan, A. A. Aczel, L. Li, M. B. Stone, G. E. Granroth, M. D. Lumsden, Y. Yiu, J. Knolle, S. Bhattacharjee, D. L. Kovrizhin, R. Moessner, D. A. Tennant, D. G. Mandrus, and S. E. Nagler, Nat. Mater. 15, 733 (2016).

[28] R. Coldea, D. A. Tennant, and Z. Tylczynski, Phys. Rev. B 68, 134424 (2003).

[29] G. Baskaran, S. Mandal, and R. Shankar, Phys. Rev. Lett. 98, 247201 (2007).

[30] J. Knolle, D. L. Kovrizhin, J. T. Chalker, and R. Moessner, Phys. Rev. Lett. 112, 207203 (2014).

[31] J. Knolle, D. L. Kovrizhin, J. T. Chalker, and R. Moessner, Phys. Rev. B 92, 115127 (2015).

[32] J. Nasu, J. Knolle, D. L. Kovrizhin, Y. Motome, and R. Moessner, Nat. Phys. 12, 912 (2016).

[33] F. Ye, S. X. Chi, H. B. Cao, B. C. Chakoumakos, J. A. Fernandez-Baca, R. Custelcean, T. F. Qi, O. B. Korneta, and G. Cao, Phys. Rev. B 85, 180403 (2012).

[34] S. K. Choi, R. Coldea, A. N. Kolmogorov, T. Lancaster, I. I. Mazin, S. J. Blundell, P. G. Radaelli, Y. Singh, P. Gegenwart, K. R. Choi, S.-W. Cheong, P. J. Baker, C. Stock, and J. Taylor, Phys. Rev. Lett. 108, 127204 (2012).

[35] J. M. Fletcher, W. E. Gardner, A. C. Fox, and G. Topping, Journal of the Chemical Society A, Inorganic, physical, theoretical, 1038 (1967).

[36] J. A. Sears, M. Songvilay, K. W. Plumb, J. P. Clancy, Y. Qiu, Y. Zhao, D. Parshall, and Y.-J. Kim, Phys. Rev. B 91, 144420 (2015).

[37] R. D. Johnson, S. C. Williams, A. A. Haghighirad, J. Singleton, V. Zapf, P. Manuel, I. I. Mazin, Y. Li, H. O. Jeschke, R. Valentí, and R. Coldea, Phys. Rev. B 92, 235119 (2015).

[38] H. B. Cao, A. Banerjee, J.-Q. Yan, C. A. Bridges, M. D. Lumsden, D. G. Mandrus, D. A. Tennant, B.C. Chakoumakos, and S. E. Nagler, Phys. Rev. B 93, 134423 (2016). 
[39] I. Kimchi and Y.-Z. You, Phys. Rev. B 84, 180407 (2011).

[40] I. I. Mazin, H. O. Jeschke, K. Foyevtsova, R. Valentí, and D. I. Khomskii, Phys. Rev. Lett. 109, 197201 (2012).

[41] J. Chaloupka, G. Jackeli, and G. Khaliullin, Phys. Rev. Lett. 110, 097204 (2013).

[42] J. G. Rau, E. K. -H. Lee, and H.-Y. Kee, Phys. Rev. Lett. 112, 077204 (2014).

[43] Y. Yamaji, Y. Nomura, M. Kurita, R. Arita, and M. Imada, Phys. Rev. Lett. 113, 107201 (2014).

[44] Y. Sizyuk, C. Price, P. Wölfle, and N. B. Perkins, Phys. Rev. B 90, 155126 (2014).

[45] S. M. Winter, Y. Li, H. O. Jeschke, and R. Valentí, Phys. Rev. B 93, 214431 (2016).

[46] H.-S. Kim and H.-Y. Kee, Phys. Rev. B 93, 155143 (2016).

[47] Y. Sizyuk, P. Wölfle, and N. B. Perkins, Phys. Rev. B 94, 085109 (2016).

[48] W. Wang, Z.-Y. Dong, S.-L. Yu, and J.-X. Li, Phys. Rev. B 96, 115103 (2017).

[49] A. Banerjee, J. Q. Yan, J. Knolle, C. A. Bridges, M. B. Stone, M. D. Lumsden, D. G. Mandrus, D. A. Tennant, R. Moessner, and S. E. Nagler, Science 356, 1055 (2017).

[50] I. A. Leahy, C. A. Pocs, P. E. Siegfried, D. Graf, S.-H. Do, K.-Y. Choi, B. Normand, and M. Lee, Phys. Rev. Lett. 118, 187203 (2017).
[51] S.-H. Baek, S.-H. Do, K.-Y. Choi, Y. S. Kwon, A. U. B. Wolter, S. Nishimoto, J. van den Brink, and B. Büchner, Phys. Rev. Lett. 119, 037201 (2017).

[52] L. Janssen, E. C. Andrade, and M. Vojta, Phys. Rev. Lett. 117, 277202 (2016).

[53] R. Yadav, N. A. Bogdanov, V. M. Katukuri, S. Nishimoto, J. van den Brink, and L. Hozoi, Sci. Rep. 6, 37925 (2016).

[54] K. J. Ran, J. H. Wang, W. Wang, Z.-Y. Dong, X. Ren, S. Bao, S. C. Li, Z. Ma, Y. Gan, Y. T. Zhang, J. T. Park, G. C. Deng, S. Danilkin, S.-L. Yu, J.-X. Li, and J. S. Wen, Phys. Rev. Lett. 118, 107203 (2017).

[55] See the Supplemental Material at http://link.aps.org/ supplemental/10.1103/PhysRevLett.119.227208 for details.

[56] A. Abragam, Principles of Nuclear Magnetism (Oxford University Press, Oxford, 1961).

[57] H. Monien and D. Pines, Phys. Rev. B 41, 6297 (1990).

[58] B. Dóra and F. Simon, Phys. Rev. Lett. 102, 197602 (2009).

[59] N. A. Fortune, S. T. Hannahs, Y. Yoshida, T. E. Sherline, T. Ono, H. Tanaka, and Y. Takano, Phys. Rev. Lett. 102, 257201 (2009).

[60] X.-Y. Song, Y.-Z. You, and L. Balents, Phys. Rev. Lett. 117, 037209 (2016)

[61] Z.-X. Liu and B. Normand, arXiv:1709.07990. 